Article

\title{
Inhibitory Effects of Resveratrol on the Epstein-Barr Virus Lytic Cycle
}

\section{Ching-Yi Yiu ${ }^{1}$, Shih-Ying Chen ${ }^{2}$, Li-Kwan Chang ${ }^{3}$, Ya-Fang Chiu ${ }^{4}$ and Tsuey-Pin Lin ${ }^{5, *}$}

1 Department of Otolaryngology, Chi Mei Medical Center, Liouying; Tainan, 717, Taiwan

2 Department of Applied Life Science and Health, Chia-Nan University of Pharmacy and Science, 60 Erh-Jen Road, Sec. 1, Jen-Te Hsiang, Tainan, 717, Taiwan

3 Institute of Microbiology and Biochemistry, National Taiwan University, Taipei, 106, Taiwan

4 Molecular Genetics Laboratory, Department of Microbiology and Immunology, Chang-Gung University, Taoyuan, 333, Taiwan

5 Department of Health and Nutrition, Chia-Nan University of Pharmacy and Science, 60 Erh-Jen Road, Sec. 1, Jen-Te Hsiang, Tainan, 717, Taiwan

* Author to whom correspondence should be addressed; E-Mail: tplin007@mail.chna.edu.tw; Fax: +886-6-2667327.

Received: 7 September 2010; in revised form: 11 October 2010 / Accepted: 13 October 2010 / Published: 14 October 2010

\begin{abstract}
Reactivation of Epstein-Barr virus (EBV) from latency to the lytic cycle is required for the production of viral particles. Here, we examine the capacity of resveratrol to inhibit the EBV lytic cycle. Our results show that resveratrol inhibits the transcription of EBV immediate early genes, the expression of EBV lytic proteins, including Rta, Zta, and EA-D and reduces viron production, suggesting that this compound may be useful for preventing the proliferation of the virus.
\end{abstract}

Keywords: resveratrol; antiviral activity; EBV

\section{Introduction}

Epstein-Barr virus (EBV) is a gamma-herpesvirus, which infects human lymphoid cells and epithelial cells [1]. Infection by this virus is associated with a number of human cancers, including 
Burkitt's lymphoma [2], nasopharyngeal carcinoma (NPC) [3] and Hodgkin's disease [4]. The reactivation of EBV from latency to the lytic cycle is necessary for the virus to produce virions and establish infections $[5,6]$. At the onset of the lytic cycle, EBV expresses two transcription factors, Rta and Zta, which are transcribed from BRLF1 and BZLF1, respectively [7]. These two proteins trigger an ordered cascade of the expression of viral lytic genes, including that of BMRF1 and BALF5, which encode diffused early antigen (EA-D) and DNA polymerase [8]. Rta and Zta can also activate IL-6 transcription in lytically-infected B cells, which leads to immortalized B cells [9]. Therefore, to reduce the disease risk and to improve the clinical outcome an effective strategy to block the viral lytic cycle is of value. Earlier studies have established that lytic EBV replication is inhibited by acyclovir and ganciclovir, which specifically inhibit the function of viral-encoded DNA polymerase [10]. An earlier study showed that epigallocatechin gallate (EGCG) inhibits the expression of the EBV immediate-early genes transcription at concentration $50 \mu \mathrm{M}$ [11]. Ethanolic extract from Andrographis paniculata and andrograpolide also inhibit the expression of Rta, Zta and EA-D at $25 \mu \mathrm{g} / \mathrm{mL}$ and $5 \mu \mathrm{g} / \mathrm{mL}$, respectively [12].

Resveratrol is a non-flavonoid polyphenol compound present in many plants and fruits. This compound has a high bioactivity and its cytoprotective action has been demonstrated. Previous studies showed that resveratrol inhibits the replication of human cytomegalovirus (HCMV) [13], herpes simplex virus type 1 (HSV-1) [14] and Varicella-zoster virus (VZV) [15]. Furthermore, resveratrol also has been reported to inhibit the expression of EBV early antigen induced by 12-Otetradecanoylphorbol 13-acetate (TPA) in Raji cells [16]. However, no preexisting study has been reported on resveratrol mediated the inhibition of EBV lytic cycle. Thus, the purpose of our study was to evaluate whether resveratrol inhibits the transcription of EBV immediate early genes, the expression of EBV lytic proteins, including Rta, Zta, and EA-D and viron production.

\section{Results and Discussion}

\subsection{Determining the toxicity of resveratrol to P3HR1 cells}

Resveratrol was added into $1 \times 10^{5}$ cells/mL P3HR1 cells. After 24 h of treatment, cell viability was determined by the MTT assay. Results showed that resveratrol exhibited no cytotoxicity toward P3HR1 cells at concentrations below $55 \mu \mathrm{M}$ (Figure 1).

\subsection{Resveratrol inhibit the expression of viral lytic proteins}

Resveratrol was added to P3HR1 cells $\left(3 \times 10^{6}\right)$ that had been treated with sodium butyrate to activate the EBV lytic cycle. At $24 \mathrm{~h}$ after lytic induction, the expression of Rta, Zta and EA-D was examined by an immunofluorescence assay which showed that at $13.8 \mu \mathrm{M}$, resveratrol reduced the expression of Rta, Zta and EA-D (Figure 2). At $27.5 \mu \mathrm{M}$, the expression of Zta and EA-D was substantially reduced and no expression of Rta was observed (Figure 2). When the concentration of resveratrol increased to $55 \mu \mathrm{M}$, no expression of Rta and Zta was observed and only few cells expressed EA-D (Figure 2). 
Figure 1. Effect of resveratrol on cell viability.

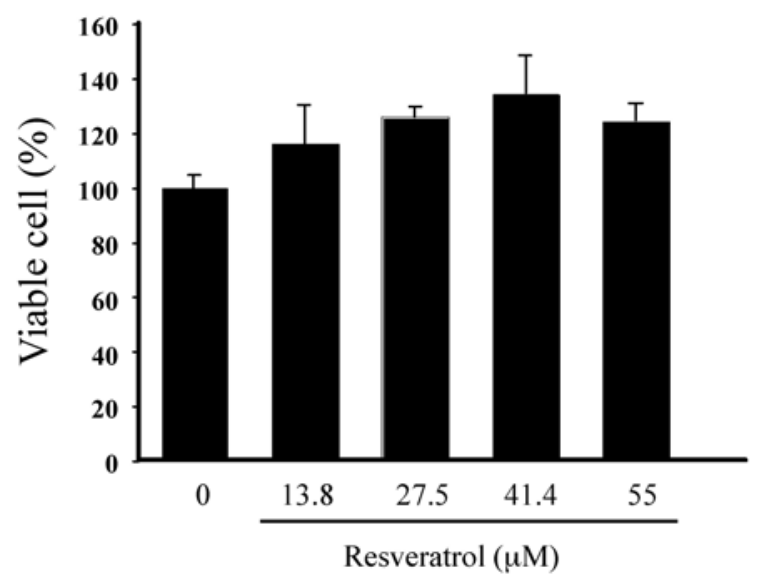

P3HR1 cells $\left(1 \times 10^{5} \mathrm{cell} / \mathrm{mL}\right)$ were treated with resveratrol. Cell numbers and viability were monitored by MTT assay after 24 h of treatment. $* p<0.05 v s$. Control.

Figure 2. Indriect immunofluorescence analysis of inhibition effects on the expression of EBV lytic genes.

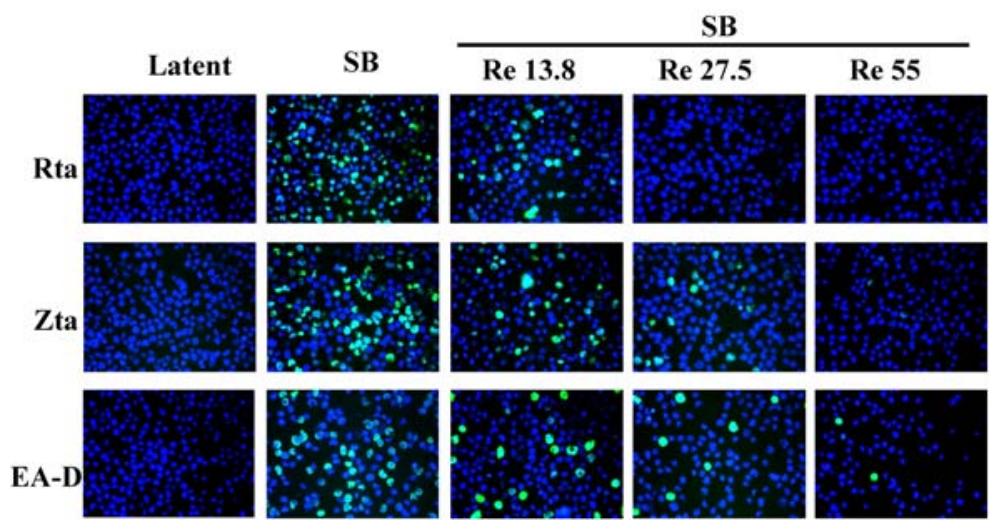

P3HR1 cells untreated with sodium butyrate (SB) and resveratrol were used as a negative control (Latent). Cells were treated with resveratrol $(\mathrm{Re})(0,13.8,27.5$ and $55 \mu \mathrm{M})$ before SB induction for $24 \mathrm{~h}$. Cells were then harvested and the expression of the lytic EBV proteins were assessed by immunofluoresence. The following primary antibodies were used: mouse monoclonal anti-Rta, anti-Zta, and anti-EA-D antibodies. The secondary antibodies were used: Alexa Fluor 488-conjugated goat anti-mouse IgG. DNA was visualized by DAPI staining; cells were examined under a fluorescence microscope.

\subsection{Flow cytometric analysis of the expression of EBV lytic proteins}

The presence of EBV lytic proteins in P3HR1 cells was further analyzed by flow cytometric analysis. The P3HR1 populations not treated with SB that expressed Rta, Zta and EA-D wwere 4.5\%, $2.1 \%$ and $4.9 \%$, respectively (Figure 3 ). The populations that expressed the three proteins after SB treatment increased to $51.2 \%, 61.4 \%$ and $46.8 \%$, respectively. After treating the cells with $13.8 \mu \mathrm{M}$ of resveratrol, the populations that expressed Rta, Zta and EA-D decreased to 29.6\%, 37.0\% and 29.6\%, 
respectively (Figure 3A). The populations that expressed Rta, Zta, and EA-D further decreased when the concentration of resveratrol was increased to $27.5 \mu \mathrm{M}$ and $55 \mu \mathrm{M}$. At $27.5 \mu \mathrm{M}$, the populations that expressed Rta, Zta, and EA-D decreased to $15.4 \%$, 20.2\%, and 17.9\%, respectively (Figure 3). At $55 \mu \mathrm{M}$, the population that expressed Rta was reduced from $51.2 \%$ to $4.7 \%$ (Figure 3); the population that expressed Zta, from $61.4 \%$ to $5.6 \%$; and the population that expressed EA-D from $46.8 \%$ to $4.5 \%$, respectively (Figure 3). These results show that resveratrol inhibited the expression of Rta, Zta and EA-D in a dose-dependent manner (Figure 3B).

Figure 3. Inhibition of the expression of EBV lytic proteins by resveratrol.

A

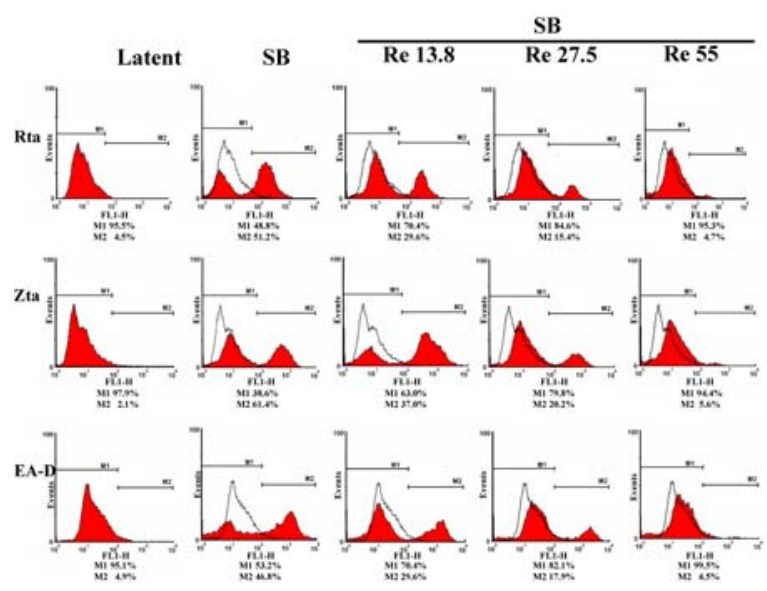

B

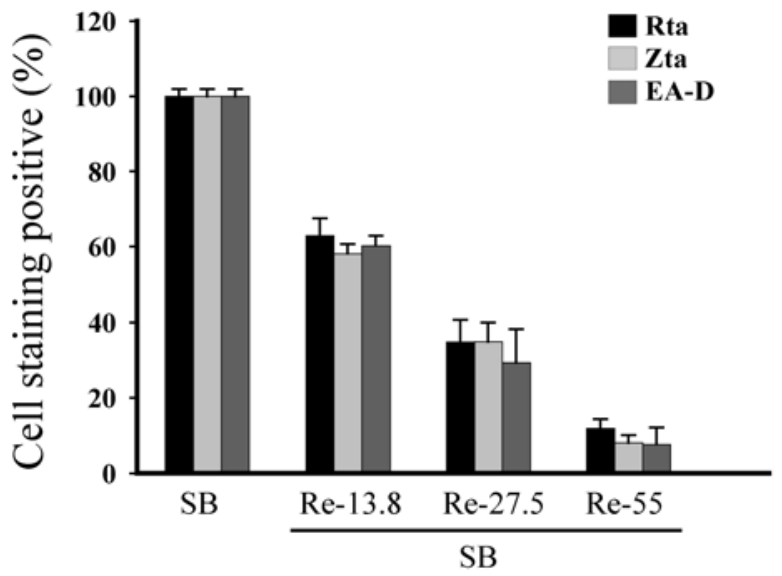

P3HR1 cells were untreated with SB and resveratrol (Latent) or treated with resveratrol (Re) (0, 13.8, 27.5 and $55 \mu \mathrm{M}$ ) before SB induction (A). At $24 \mathrm{~h}$ after treatment, cells were stained with primary mouse monoclonal antibodies (anti-Rta or anti-Zta or anti-EA-D) and Alexa Fluor 488-conjugated goat anti-mouse IgG and then analyzed by flow cytometry. M2 represents fluorescence stained cells. The number of cells expressing EBV lytic proteins was quantified by averaging the results from three experiments (B). The data are presented as means with standard deviation. 


\subsection{Inhibiting the transcription of EBV immediate-early genes}

The activity of the BRLF1 and BZLF1 promoters was analyzed by transient transfection assay in P3HR1 cells using the reporter plasmids pRLUC and pZLUC, respectively. Results showed that resveratrol at $6.9 \mu \mathrm{M}$ inhibited the activity of BRLF1 and BZLF1 promoters by $56 \%$ and $83 \%$. Resveratrol at 13.8 $\mu \mathrm{M}$ decreased the BRLF1 and BZLF1 promoters' activity to background (Figure 4).

Figure 4. Inhibition of the transcription of the BRLF1 and BZLF1 promoters by resveratrol.

A

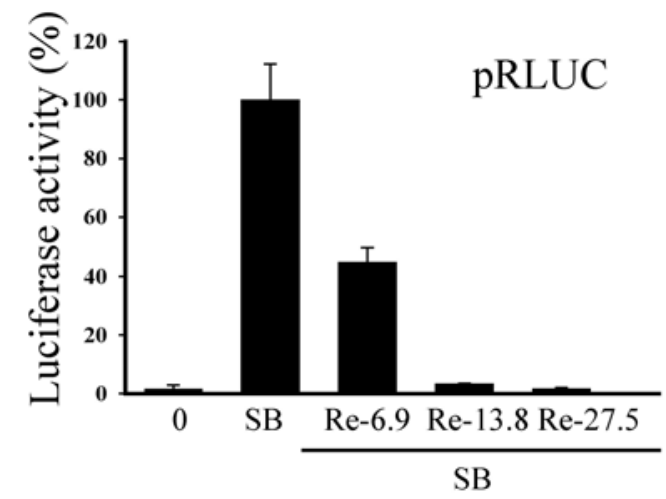

B

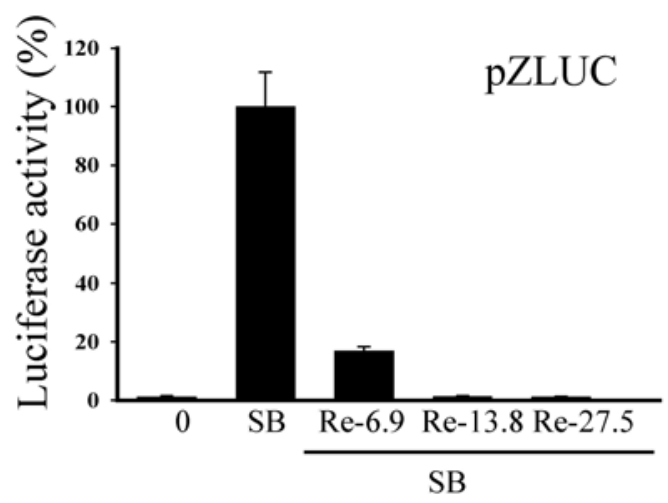

P3HR1 cells were transfected with pRLUC (A) or pZLUC (B) and treated with resveratrol (Re) (0, 6.9, 13.8 and $27.5 \mu \mathrm{M}$ ) before lytic induction (SB). At $24 \mathrm{~h}$ after transfection, luciferase activity was measured using a luminometer. Each transfection experiment was performed three times, and each sample in the experiment was prepared in duplicate.

\subsection{Inhibition of the production of EBV particles}

P3HR1 cells were then treated with 13.8, 27.5 and $55 \mu \mathrm{M}$ of resveratrol after lytic induction. After culturing for five days, EBV particles released into medium were isolated. Real-time qPCR showed that resveratrol at a concentration of $27.5 \mu \mathrm{M}$ decreased virus production by $42 \%$ (Figure 5). Resveratrol at aconcentration of $55 \mu \mathrm{M}$ reduced the production of EBV particles by $74 \%$ (Figure 5 ).

Previous studies showed that lytic EBV proteins actually induce the expression of B-cell growth factor, IL-6, cellular IL-10 and viral IL-10, allowing B cells to grow more efficiently $[17,18]$. Lytically-infected cells also produce VEGF and thus contribute to angiogenesis in both B-cell and epithelial-cell malignancies [18]. Therefore, new treatment strategies aimed at completely suppressing the expression of all lytic viral proteins is usefully in controlling early EBV-associated malignancies. This study finds that resveratrol significantly reduces the expression of EBV immediate-early proteins, Rta, Zta and EA-D in a dose-dependent manner (Figure 2 and 3). In other words, resveratrol interferes with an early step of EBV replication cycle. The concentration of resveratrol needed to inhibit EBV immediate-early proteins expression by $50 \%\left(\mathrm{EC}_{50}\right)$ is approximately $24 \mu \mathrm{M}$. The cytotoxicity of resveratrol was determined by the MTT assay and resveratrol under the concentration of $55 \mu \mathrm{M}$ did not affect the P3HR1 cells viability. Earlier study showed that EGCG inhibits the expression of EBV lytic proteins at concentration $50 \mu \mathrm{M}$ [11]. The concentration of glycyrrhizic acid required to inhibit EBV 
EA/VCA expression by $50 \%$ is $42 \mu \mathrm{M}$ [19]. These result suggested that resveratrol is more effective than EGCG and glycyrrhizic acid in inhibiting the expression of EBV immediate-early genes.

Figure 5. Resveratrol treatment and production of EBV particles.

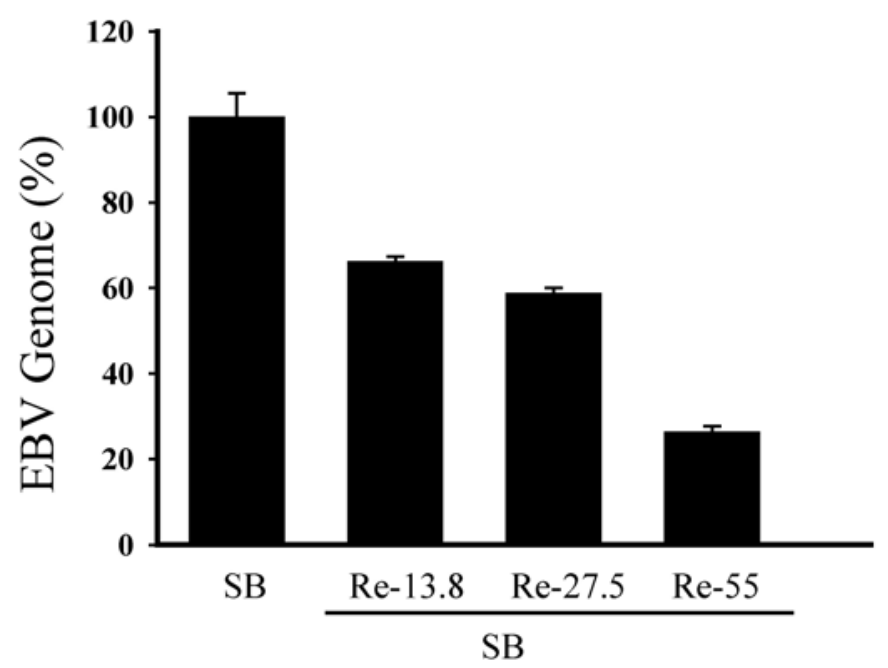

P3HR1 cells were treated with resveratrol $(\mathrm{Re})(0,13.8,27.5$ and $55 \mu \mathrm{M})$ before SB induction. Five day after the treatment, EBV particles were harvested by centrifugation. The amounts of EBV DNA isolated from the particles were determined by real-time qPCR. The copy number of EBV genome was calculated by using maxi-EBV. EBV Genome (\%) = Log of copy number (Re treated before SB induction - Re and SB untreated)/log of copy number (SB treated only - Re and SB untreated) $* 100$. The data are presented as mean \pm S.D. from at least three independent experiments.

Our study also demonstrates that resveratrol inhibits the transcriptional activity of BRLF1 and BZLF1 promoters, in turn, affect viral lytic proteins expression. Moreover, the inhibition actually decreases the production of mature viral particles. Real-time qPCR indicates that the effective concentration of resveratrol required to inhibit EBV genome copy numbers by $50 \%\left(\mathrm{EC}_{50}\right)$ is $52.2 \mu \mathrm{M}$ (Figure 5). The $\mathrm{EC}_{50}$ values of resveratrol obtained by real-time qPCR is approximately $52.2 \mu \mathrm{M}$, in contrast to the $24 \mu \mathrm{M}$ value determined by flow cytometry. However, the inhibitory profile remains unchanged, indicating that flow cytometry is as reliable as the real-time qPCR method for initial drug screening.

The molecular mechanism underlying the inhibition of EBV early gene expression by resveratrol is unclear. Previous studies showed that resveratrol inhibits the activation of p38 MAPK, ERK and JNK signaling and affects the activation of the promoters that are activated by AP-1 or ATF2 [20]. Because both the BRLF1 and BZLF1 promoters are strongly activated by AP-1 and ATF2 [21-23]. The inhibition of the activation of signaling pathways may explain how resveratrol inhibits the EBV lytic cycle. Resveratrol also has been reported to activate the deacetylase activity of sirtuin protein [24]. Histone acetylation at the BRLF1 promoter allows the virus to express Rta and to activate the viral lytic cycle [25]. Moreover, Zta-directed nucleosome acetylation activates the transcriptional activity of viral immediate-early gene [26]. Thus, the inhibition of resveratrol on deacetylase activity of sirtuin protein may involve in the transcriptional inhibition of BRLF1 and BZLF1 promoters. 


\section{Experimental}

\subsection{Compound}

Resveratrol (Sigma, St. Louis, MO, USA) was dissolved in dimethylsulfoxide (DMSO) before use.

\subsection{Cell culture and lytic induction of EBV}

P3HR1, a Burkitt's lymphoma cell line which is latently infected by EBV, was cultured in RPMI 1640 medium containing 10\% fetal calf serum (Biological Industries, Beit Haemek, Israel). Cells were treated with $3 \mathrm{mM}$ of sodium butyrate (SB) to induce the EBV lytic cycle [27].

\subsection{Cell viability assay}

A $1 \mathrm{mg} / \mathrm{mL}$ solution of [3-(4,5-dimethyldiazol-2-yl)-2,5-diphenyltetrazolium bromide (MTT) in RPMI 1640 medium was added to $1 \times 10^{5}$ P3HR1 cells. The dehydrogenase activity of the viable cells was measured using the method of Carmichael et al. [28].

\subsection{Indirect immunofluorescence staining}

Immunofluoresence staining was performed as described [12], using mouse monoclonal anti-Rta, anti-Zta (Argene, Varilhes, France), and anti-EA-D antibodies (Chemicon, Temecula, CA, USA). Secondary antibodies used in the study included Alexa Fluor 488-conjugated goat anti-mouse IgG from Invitrogen. DNA was visualized by staining with 4',6'-diamido-2-phenylindole (DAPI). Cells were examined under the Axioskop 2 plus fluorescence microscope (Zeiss, Oberkochen, Germany).

\subsection{Flow cytometric analysis}

P3HR1 cells were treated with antibodies as described for indirect immunofluorescence analysis and fixed in 4\% paraformaldehyde. Finally, cells were resuspended in 1\% paraformaldehyde and analyzed using a flow cytometer (model FACScanTO, BD Biosciences, New Jersey, USA).

\subsection{Transient transfection and luciferase assays}

P3HR1 cells $\left(5 \times 10^{6}\right)$ were transfected with $10 \mu \mathrm{g}$ of pRLUC and pZLUC with a Bio-Rad electroporator using the method of Chang et al. [25,29]. Cells were harvested at $24 \mathrm{~h}$ after transfection and luciferase activity was determined using a luminometer (Berthold, Bad Wildbad, Germany) according to a method described elsewhere [25]. 


\subsection{Quantification of EBV particles produced by P3HR1 cells}

P3HR1 cells were cultured for 5 days. EBV particles released into the culture medium were harvested by centrifugation. Viral DNA was extracted and the amount of EBV DNA was determined by real-time PCR using an iCycleriQ multicolor real-time PCR detection system (BioRad, CA, USA) with primer and a probe that were specific to the EBNA1 gene [30]. The copy number of the EBV genome was calculated using maxi-EBV DNA extracted from E. coli as a reference. The molecular weight of maxi-EBV is about $1.2 \times 10^{7}$ and $1 \mathrm{ng}$ of maxi-EBV equals to $5.05 \times 10^{6}$ copies of the maxi-EBV genome.

\subsection{Statistical analysis}

Data were analyzed statistically by one-way analysis of variance (ANOVA) using the SAS JMP 6.0 software package. Data are presented as means \pm S.D. and a $p$ value of $<0.05$ was regarded as significant.

\section{Conclusions}

To summarize the whole study, our results clearly demonstrate that resveratrol inhibits the transcription of lytic genes and the lytic cycle of EBV to reduce the production of viral particles. Resveratrol could be of potential use for the development of anti-EBV drugs.

\section{Acknowledgements}

This work was supported by research grants 96-2320-B-041-009 from the National Science Council, R.O.C., and CLFHR9620 from Chi Mei Medical Center, Liouying, Tainan, Taiwan.

\section{References}

1. Roizman, B.; Carmichael, L.E.; Deinhardt, F.; de-The, G.; Nahmias, A.J.; Plowright, W.; Rapp, F.; Sheldrick, P.; Takahashi, M.; Wolf, K. Herpesviridae. Definition, provisional nomenclature, and taxonomy. The Herpesvirus Study Group, the International Committee on Taxonomy of Viruses. Intervirology 1981, 16, 201-217.

2. Magrath, I.; Jain, V.; Bhatia, K. Epstein-Barr virus and Burkitt's lymphoma. Semin. Cancer Biol. 1992, 3, 285-295.

3. zur Hausen, H.; Schulte-Holthausen, H.; Klein, G.; Henle, W.; Henle, G.; Clifford, P.; Santesson, L. EBV DNA in biopsies of Burkitt tumours and anaplastic carcinomas of the nasopharynx. Nature 1970, 228, 1056-1058.

4. Weiss, L.M.; Movahed, L.A.; Warnke, R.A.; Sklar, J. Detection of Epstein-Barr viral genomes in Reed-Sternberg cells of Hodgkin's disease. N. Engl. J. Med. 1989, 320, 502-506.

5. Schwarzmann, F.; Jager, M.; Prang, N.; Wolf, H. The control of lytic replication of Epstein-Barr virus in B lymphocytes (Review). Int. J. Mol. Med. 1998, 1, 137-142. 
6. Hopwood, P.A.; Brooks, L.; Parratt, R.; Hunt, B.J.; Bokhari, M.; Thomas, J.A.; Yacoub, M.; Crawford, D.H. Persistent Epstein-Barr virus infection: unrestricted latent and lytic viral gene expression in healthy immunosuppressed transplant recipients. Transplantation 2002, 74, 194-202.

7. Giot, J.F.; Mikaelian, I.; Buisson, M.; Manet, E.; Joab, I.; Nicolas, J.C.; Sergeant, A. Transcriptional interference between the EBV transcription factors EB1 and R: both DNA-binding and activation domains of EB1 are required. Nucleic Acids Res. 1991, 19, 1251-1258.

8. Fixman, E.D.; Hayward, G.S.; Hayward, S.D. trans-acting requirements for replication of Epstein-Barr virus ori-Lyt. J. Virol. 1992, 66, 5030-5039.

9. Tosato, G.; Tanner, J.; Jones, K.D.; Revel, M.; Pike, S.E. Identification of interleukin-6 as an autocrine growth factor for Epstein-Barr virus-immortalized B cells. J. Virol. 1990, 64, 3033-3041.

10. Meerbach, A.; Holy, A.; Wutzler, P.; De Clercq, E.; Neyts, J. Inhibitory effects of novel nucleoside and nucleotide analogues on Epstein-Barr virus replication. Antivir. Chem. Chemother. 1998, 9, 275-282.

11. Chang, L.K.; Wei, T.T.; Chiu, Y.F.; Tung, C.P.; Chuang, J.Y.; Hung, S.K.; Li, C.; Liu, S.T. Inhibition of Epstein-Barr virus lytic cycle by (-)-epigallocatechin gallate. Biochem. Biophys. Res. Commun. 2003, 301, 1062-1068.

12. Lin, T.P.; Chen, S.Y.; Duh, P.D.; Chang, L.K.; Liu, Y.N. Inhibition of the epstein-barr virus lytic cycle by andrographolide. Biol. Pharm. Bull. 2008, 31, 2018-2023.

13. Evers, D.L.; Wang, X.; Huong, S.M.; Huang, D.Y.; Huang, E.S. 3,4',5-Trihydroxy-trans-stilbene (resveratrol) inhibits human cytomegalovirus replication and virus-induced cellular signaling. Antivir. Res. 2004, 63, 85-95.

14. Docherty, J.J.; Fu, M.M.; Stiffler, B.S.; Limperos, R.J.; Pokabla, C.M.; DeLucia, A.L. Resveratrol inhibition of herpes simplex virus replication. Antivir. Res. 1999, 43, 145-155.

15. Docherty, J.J.; Sweet, T.J.; Bailey, E.; Faith, S.A.; Booth, T. Resveratrol inhibition of varicella-zoster virus replication in vitro. Antivir. Res. 2006, 72, 171-177.

16. Kapadia, G.J.; Azuine, M.A.; Tokuda, H.; Takasaki, M.; Mukainaka, T.; Konoshima, T.; Nishino, H. Chemopreventive effect of resveratrol, sesamol, sesame oil and sunflower oil in the Epstein-Barr virus early antigen activation assay and the mouse skin two-stage carcinogenesis. Pharmacol. Res. 2002, 45, 499-505.

17. Jones, R.J.; Seaman, W.T.; Feng, W.H.; Barlow, E.; Dickerson, S.; Delecluse, H.J.; Kenney, S.C. Roles of lytic viral infection and IL-6 in early versus late passage lymphoblastoid cell lines and EBV-associated lymphoproliferative disease. Int. J. Cancer 2007, 121, 1274-1281.

18. Hong, G.K.; Kumar, P.; Wang, L.; Damania, B.; Gulley, M.L.; Delecluse, H.J.; Polverini, P.J.; Kenney, S.C. Epstein-Barr virus lytic infection is required for efficient production of the angiogenesis factor vascular endothelial growth factor in lymphoblastoid cell lines. J. Virol. 2005, 79, 13984-13992.

19. Lin, J.C.; Cherng, J.M.; Hung, M.S.; Baltina, L.A.; Baltina, L.; Kondratenko, R. Inhibitory effects of some derivatives of glycyrrhizic acid against Epstein-Barr virus infection: structure-activity relationships. Antivir. Res. 2008, 79, 6-11. 
20. Kang, O.H.; Jang, H.J.; Chae, H.S.; Oh, Y.C.; Choi, J.G.; Lee, Y.S.; Kim, J.H.; Kim, Y.C.; Sohn, D.H.; Park, H.; Kwon, D.Y. Anti-inflammatory mechanisms of resveratrol in activated HMC-1 cells: pivotal roles of NF-kappaB and MAPK. Pharmacol. Res. 2009, 59, 330-337.

21. Matusali, G.; Arena, G.; De Leo, A.; Di Renzo, L.; Mattia, E. Inhibition of p38 MAP kinase pathway induces apoptosis and prevents Epstein Barr virus reactivation in Raji cells exposed to lytic cycle inducing compounds. Mol. Cancer 2009, 8, 18.

22. Rahman, M.M.; Kukita, A.; Kukita, T.; Shobuike, T.; Nakamura, T.; Kohashi, O. Two histone deacetylase inhibitors, trichostatin A and sodium butyrate, suppress differentiation into osteoclasts but not into macrophages. Blood 2003, 101, 3451-3459.

23. Adamson, A.L.; Darr, D.; Holley-Guthrie, E.; Johnson, R.A.; Mauser, A.; Swenson, J.; Kenney, S. Epstein-Barr virus immediate-early proteins BZLF1 and BRLF1 activate the ATF2 transcription factor by increasing the levels of phosphorylated p38 and c-Jun N-terminal kinases. J. Virol. 2000, 74, 1224-1233.

24. Borra, M.T.; Smith, B.C.; Denu, J.M. Mechanism of human SIRT1 activation by resveratrol. J. Biol. Chem. 2005, 280, 17187-17195.

25. Chang, L.K.; Liu, S.T. Activation of the BRLF1 promoter and lytic cycle of Epstein-Barr virus by histone acetylation. Nucleic Acids Res. 2000, 28, 3918-3925.

26. Deng, Z.; Chen, C.J.; Chamberlin, M.; Lu, F.; Blobel, G.A.; Speicher, D.; Cirillo, L.A.; Zaret, K.S.; Lieberman, P.M. The CBP bromodomain and nucleosome targeting are required for Zta-directed nucleosome acetylation and transcription activation. Mol. Cell Biol. 2003, 23, 2633-2644.

27. Luka, J.; Kallin, B.; Klein, G. Induction of the Epstein-Barr virus (EBV) cycle in latently infected cells by n-butyrate. Virology 1979, 94, 228-231.

28. Carmichael, J.; DeGraff, W.G.; Gazdar, A.F.; Minna, J.D.; Mitchell, J.B. Evaluation of a tetrazolium-based semiautomated colorimetric assay: assessment of chemosensitivity testing. Cancer Res. 1987, 47, 936-942.

29. Chang, P.J.; Chang, Y.S.; Liu, S.T. Role of Rta in the translation of bicistronic BZLF1 of Epstein-Barr virus. J. Virol. 1998, 72, 5128-5136.

30. Chiu, Y.F.; Tung, C.P.; Lee, Y.H.; Wang, W.H.; Li, C.; Hung, J.Y.; Wang, C.Y.; Kawaguchi, Y.; Liu, S.T. A comprehensive library of mutations of Epstein Barr virus. J. Gen. Virol. 2007, 88, 2463-2472.

Sample Availability: Sample of the compound is available from the authors.

(C) 2010 by the authors; licensee MDPI, Basel, Switzerland. This article is an open access article distributed under the terms and conditions of the Creative Commons Attribution license (http://creativecommons.org/licenses/by/3.0/). 